\title{
Surgical treatment for acromioclavicular joint osteoarthritis: patient selection, surgical options, complications, and outcome
}

\author{
Salvatore Docimo Jr. • Dellene Kornitsky • \\ Bennett Futterman · David E. Elkowitz
}

Published online: 11 March 2008

(C) Humana Press 2008

\begin{abstract}
Osteoarthritis is one of the most common causes of pain originating from the acromioclavicular (AC) joint. An awareness of appropriate diagnostic techniques is necessary in order to localize clinical symptoms to the AC joint. Initial treatments for AC joint osteoarthritis, which include non-steroidal anti-inflammatory drugs (NSAIDS) and corticosteroids, are recommended prior to surgical interventions. Distal clavicle excision, the main surgical treatment option, can be performed by various surgical approaches, such as open procedures, direct arthroscopic, and indirect arthroscopic techniques. When choosing the best surgical option, factors such as avoidance of AC ligament damage, clavicular instability, and post-operative pain must be considered. This article examines patient selection, complications, and outcomes of surgical treatment options for $\mathrm{AC}$ joint osteoarthritis.
\end{abstract}

S. Docimo Jr. ( $\square)$

Anatomy and Pathology Academic Medicine Fellow, New York College of Osteopathic Medline, Old Westbury, NY, USA

e-mail: sdocimo@nyit.edu

S. Docimo Jr. · D. E. Elkowitz

Department of Pathology, New York College of Osteopathic

Medicine, Old Westbury, NY, USA

D. E. Elkowitz

e-mail: delkowit@nyit.edu

D. Kornitsky

New York College of Osteopathic Medicine, Old Westbury, NY, USA

e-mail: dkornits@nyit.edu

B. Futterman

Department of Anatomy, New York College of Osteopathic

Medicine, Old Westbury, NY, USA

e-mail: bfutterm@nyit.edu
Keywords Acromioclavicular joint · Osteoarthritis · Arthritis · Surgery $\cdot$ Complications · Outcomes

\section{Introduction}

Shoulder pain has become the third most common cause of musculoskeletal consultation in primary care with a prevalence of self-reported shoulder pain estimated to be between $16 \%$ and $26 \%$ [1]. One of the underlying causes of these complaints is pathology of the acromioclavicular (AC) joint, with a prevalence much higher than generally realized [2]. An analysis of 1,000 patients with shoulder pain revealed AC joint abnormalities on standard radiographs to have a prevalence of $12.7 \%$ [3].

Osteoarthritis, the most common cause of shoulder pain originating from the $\mathrm{AC}$ joint, is a frequent finding in patients older than 50 years of age [4]. A study demonstrated $54-57 \%$ of elderly patients have radiographic evidence of degenerative arthritis of the AC joint [5]. Evaluation of MRIs among asymptomatic subjects demonstrated the prevalence of AC joint osteoarthritis to be between $48 \%$ and $82 \%$ [6, 7].

The treatment of AC joint pathology can be difficult considering non-invasive measures often only provide short-term benefits. Non-steroidal anti-inflammatory drugs (NSAIDS) and corticosteroid injections have shown to improve pain and function temporarily, causing patients to seek surgical treatment. One study found injection provided on an average 20 days of pain relief, with 18 of the 27 patients $(67 \%)$ seeking surgical treatment following injections [8]. A variety of surgical treatments exist, ranging from open distal clavicle resection to direct and indirect arthroscopic surgical resection. This article reviews appropriate evaluation of patients presenting with 
AC joint pain, non-surgical interventions, surgical indications, and surgical techniques.

\section{Anatomy and pathology}

The AC joint is a diarthrodial joint between the lateral portion of the clavicle and the acromion (Fig. 1). Stability of the AC joint is facilitated by the capsule, ligaments, and intra-articular disc [9]. Capsular ligaments surround the AC joint and provide stability superiorly, inferiorly, posteriorly, and anteriorly [10]. The conoid and trapezoid ligaments, which collectively comprise the coracoclavicular ligament, span the distance between the superior surface of the coracoid to the conoid tuberosity and trapezoid ridge of the clavicle and prevent vertical displacement of the AC joint [11, 12] (Fig. 2). The intra-articular disc varies in size and shape and undergoes rapid degeneration, rendering it functionally trivial by the fourth decade [9].

Primary osteoarthritis more commonly affects the AC joint than glenohumeral joint [13], while post-traumatic AC joint arthritis is even more prevalent due to the high incidence of injury to the joint [14]. Arthritic symptoms have been demonstrated in Grade I and II sprains of the AC joint in $8 \%$ and $42 \%$ of patients, respectively $[15,16]$.

Failure or absence of the intra-articular disc likely contributes to the high rate of early degenerative changes seen in the AC joint [17]. The intra-articular disc is shown to begin its natural progression of degeneration as early as the second decade of life [18]. High axial loads transferred

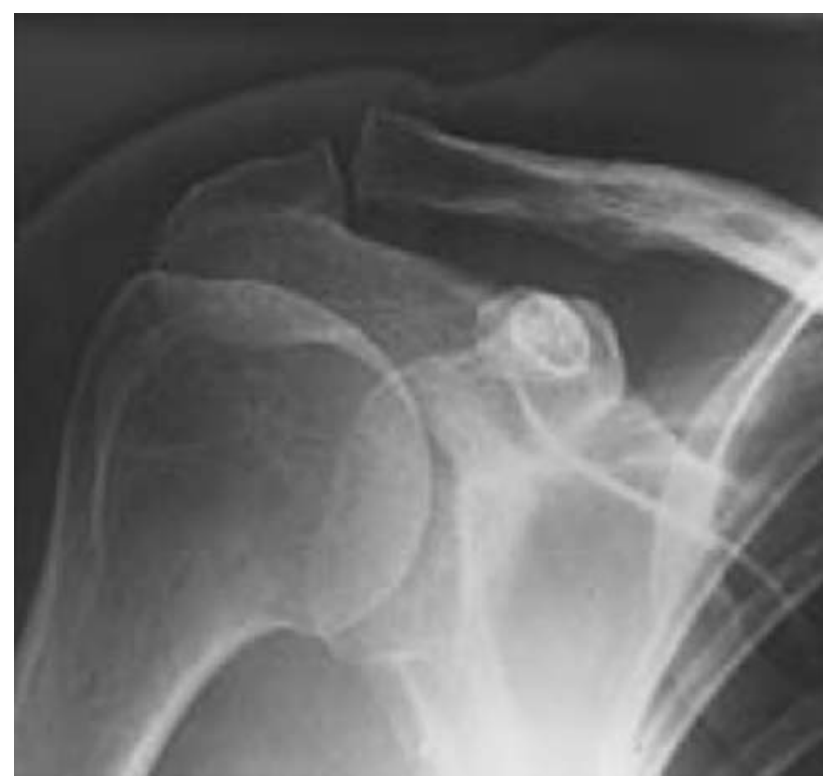

Fig. 1 Zanca view radiograph demonstrating the anatomy of the acromioclavicular joint. (C)1999 American Academy of Orthopaedic Surgeons. Reprinted from the Journal of the American Academy of Orthopaedic Surgeons, Volume 7 (3), pp. 176-188 with permission

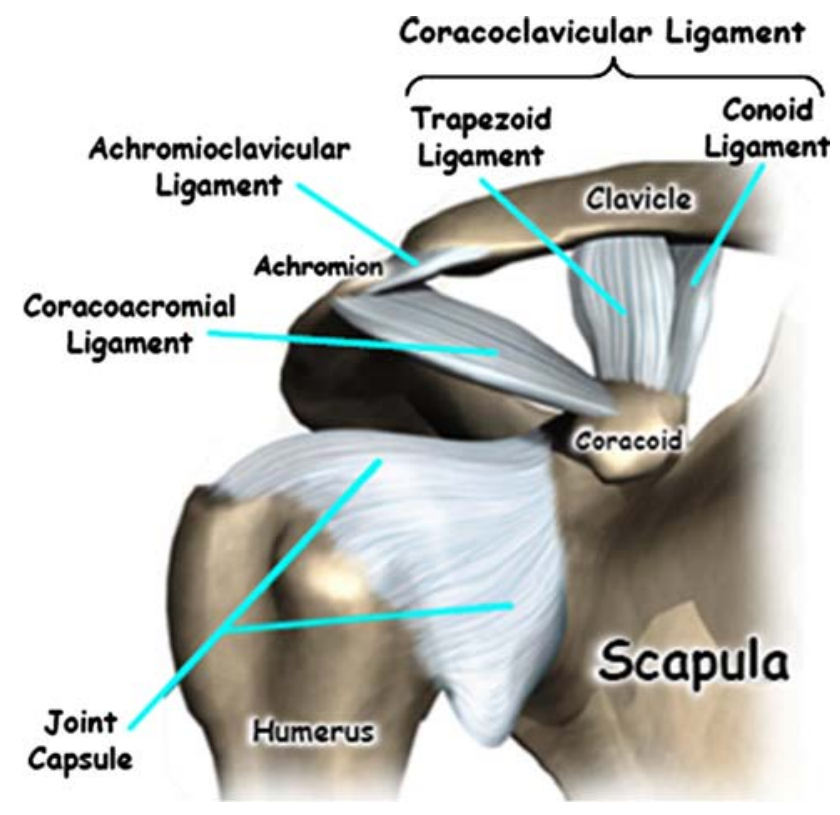

Fig. 2 Bony and ligamentous structures of the acromioclavicular joint. Image courtesy of Medical Multimedia Group LLC, www.eOrthopod.com

through the small surface area of the AC joint, which has an average joint size of $9 \times 19 \mathrm{~mm}$ in an adult, may place high stresses on the articular surface causing failure, such as osteoarthritis or osteolysis, among weightlifters [11]. High axial loads, when compounded with a degenerated or absent intra-articular disc, are even more likely to cause osteoarthritis.

\section{Patient presentation and evaluation}

A study of 21 male and 35 female patients with AC joint osteoarthritis found shoulder pain presented during the ages of 53-55 years with less than $50 \%$ of these patients reporting a history of trauma [19]. Patients often present with an intact range of motion with the exception of crossbody adduction, behind the back motions, and overhead reaching, which all produce pain localized to the $\mathrm{AC}$ joint $[13,17,20]$. However, pain to the deltoid area upon crossbody adduction has also been noted and is likely caused by irritation of the underlying subacromial bursa by inferiorly projecting osteophytes of the AC joint [17] (Fig. 3).

In addition to osteoarthritis, the differential diagnosis of AC joint pain includes calcific tendonitis, glenohumeral arthritis, adhesive capsulitis, and rotator cuff impingement syndrome [17]. Accurate diagnosis and localization of pathology to the AC joint is vital in determining the correct treatment protocol in order to avoid persistent shoulder pain. Upon physical examination, the AC joint may be tender to palpation [21]. Pain elicited by the motion of 


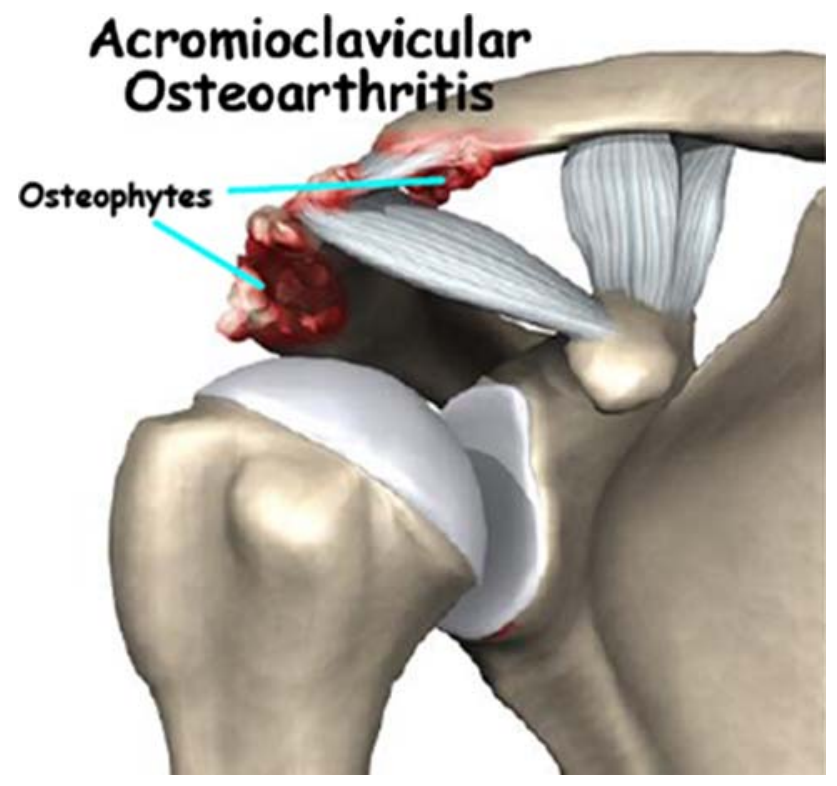

Fig. 3 Schematic demonstration the presentation of acromioclavicular osteoarthritis and location of osteophytes. Image courtesy of Medical Multimedia Group LLC, www.eOrthopod.com

forward flexion to $90^{\circ}$ with horizontal adduction (crossover test) or straight-ahead pushing (as in the bench press exercise) further suggests AC joint involvement [22] (Fig. 4).

Acromioclavicular joint involvement can be confirmed by an injection of a local anesthetic. Injection of $0.5-2 \mathrm{~mL}$ of $1 \%$ or $2 \%$ lidocaine or $0.5 \mathrm{~mL}$ of 0.25 or $0.5 \%$ bupivacaine into the $\mathrm{AC}$ joint should provide a significant reduction in symptoms [21]. A continuation of pain following anesthetic injection suggests other shoulder pathologies, most commonly rotator cuff injury [14] due to the close proximity of the AC joint to the subacromial bursa and rotator cuff [17]. Another diagnostic method to confirm the location of a pathological process involving the $\mathrm{AC}$ joint is injection of $5 \mathrm{~mL}$ of $1 \%$ lidocaine into the subacromial space, with persistence of AC joint pain following the injection [21].

Radiographs are the initial diagnostic imaging modality of choice [23], with anterior-posterior views demonstrating degenerative changes, subchondral cysts, sclerosis, osteophytes, and joint-space narrowing [17]. The Zanca view, which consists of angling the X-ray source $10-15^{\circ}$ superiorly and decreasing the kilovoltage to $50 \%$ standard exposure [17], is helpful in evaluating AC joint pathology by allowing visualization of distally projecting osteophytes of the acromion [14] (Fig. 5). Computed tomography is preferred when evaluating arthritic osseous changes of the $\mathrm{AC}$ joint such as joint narrowing, erosions, and subchondral cysts $[14,23]$. Magnetic resonance imaging has the ability to detect capsular hypertrophy, effusions, and subchondral edema [17]. A comparison of MRI findings of the

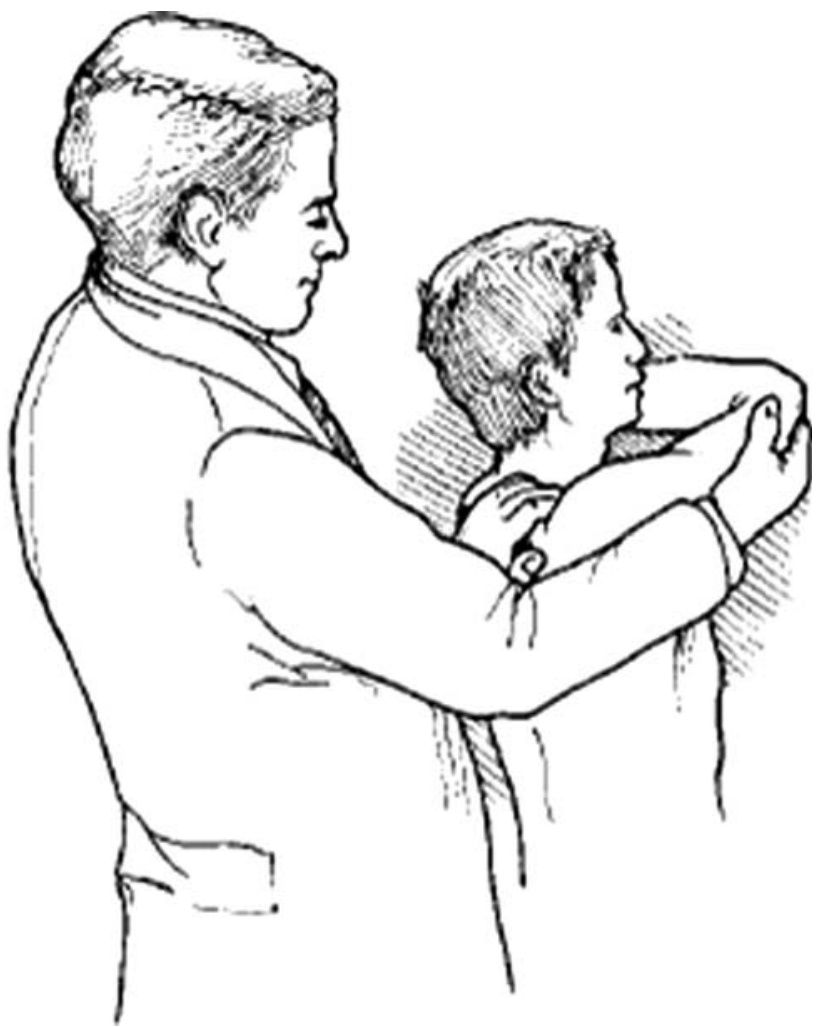

Fig. 4 The cross-over adduction test is performed by the motion of forward flexion to $90^{\circ}$ with horizontal adduction of the arm across the chest. Reproducible pain over the joint suggests AC joint involvement. (C)1999 American Academy of Orthopaedic Surgeons. Reprinted from the Journal of the American Academy of Orthopaedic Surgeons, Volume 7 (3), pp. 176-188 with permission

$\mathrm{AC}$ joint in symptomatic and asymptomatic patients correlates edema of the distal clavicle with the presence of symptoms [24]. Though ultrasound can be used to detect the presence of AC joint effusions, it cannot differentiate between effusions due to acute inflammatory processes versus degenerative changes [25], thus rendering it less effective in the evaluation of AC joint pathology [17].

\section{Non-surgical treatments}

Initial treatment of $\mathrm{AC}$ joint arthritis is non-operative and includes activity modification, physical therapy, non-steriodal anti-inflammatory medications (NSAIDs), and local AC joint injection of anesthetics or corticosteroids [26]. Activity modification includes avoidance of repetitive motions causing the pain, such as push-ups, dips, flies, and bench press exercises [17]. Physical therapy would include exercises to maintain active range of motion and increase muscle strength for scapular stabilization [26]. However, physical therapy is not as effective for AC joint arthritis as it is for rotator cuff disease [27]. 


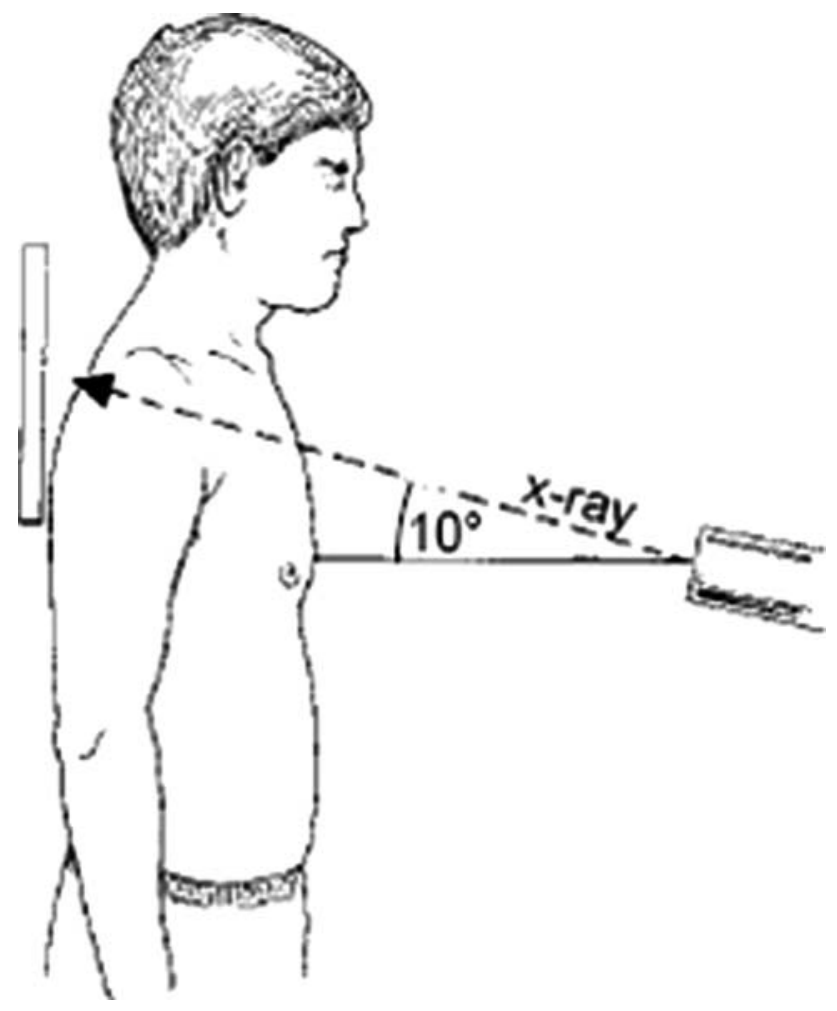

Fig. 5 The Zanca view of the AC joint, which is obtained by angling the X-ray source $10-15^{\circ}$ superiorly and decreasing the kilovoltage to $50 \%$ standard exposure, is helpful in evaluating AC joint pathology such as distally projecting osteophytes. (C)1999 American Academy of Orthopaedic Surgeons. Reprinted from the Journal of the American Academy of Orthopaedic Surgeons, Volume 7 (3), pp. 176-188 with permission

Corticosteroid injection into the $\mathrm{AC}$ joint is warranted following previous failed trials of NSAIDs and activity modification, and also if a diagnostic local anesthetic injection provides relief. The $\mathrm{AC}$ joint can be located by first palpating the soft spot where the clavicle and spine of the scapula meet and then moving slightly anterior [27]. The skin is anesthetized and the needle is inserted into the joint-space using a superior approach and moved inferiorly until a decrease in resistance is felt as the needle enters the capsule [14]. Injections of $0.25-0.5 \mathrm{~mL}$ of betamethasone sodium phosphate and acetate or $0.25-0.5 \mathrm{~mL}$ methylprednisolone, $40 \mathrm{mg} / \mathrm{mL}$ are recommended [17]. Limits of two to four injections per year with a total of twenty [17, 21] are recommended, as excessive corticosteroid administration may cause subcutaneous fat atrophy and dermal thinning [28].

In some cases the pain relief afforded by corticosteroid injection may be short in duration. Jacob and Sallay [8] followed 31 patients diagnosed with AC joint arthropathy and concluded AC joint corticosteroid injection offered short-term pain relief but did not alter natural disease progression. The 31 patients received $1 \mathrm{~mL}$ of Celestone/ Soluspan or dexamethasone and $2 \mathrm{~mL}$ of lidocaine injections. Of the 31 patients four were excluded from the study as they were lost to follow-up. The mean duration of improvement was 20 days, as reported by patients, with a range of $2 \mathrm{~h}$ to 3 months. Of the 27 patients 18 underwent distal clavicle resection at an average of 4 months postinjection. Only five of the remaining nine patients were considered to have had long-term therapeutic benefit from the injections. Of the 31 patients with AC joint arthropathy who received corticosteroid injection, 93\% reported improvement in pain and function, $81 \%$ failed to obtain long-term results, and $67 \%$ underwent distal clavicle resection. Though osteoarthritis is considered a noninflammatory process, recent evidence demonstrates a likely inflammatory component [17] which suggests corticosteroids should play a role in treatment.

\section{Surgical treatments}

Treatment selection

Surgical treatment options become apparent once all noninvasive treatment modalities have failed to provide adequate pain relief and persistent symptoms continue to interfere with activities of daily living [17, 26]. At least 6 months of conservative treatment should be attempted before surgery [17]. Variables such as patient occupation, age, degree of activity limitation, shoulder dominance, and patient goals should be considered by both patient and physician before a decision concerning surgical treatment is made [14].

Distal clavicle excision, which prevents abutment of the distal clavicle against the medial acromion [22], is the mainstay of surgical treatment for AC joint arthritis [26] (Fig. 6). Various surgical techniques, such as an open approach or direct and indirect arthroscopic approaches, are available. The open technique frequently utilizes a 3- to 5 -cm transverse or perpendicular saber skin incision with division of the deltotrapezial fascia. An oscillating saw is used to excise a 1 - to $2-\mathrm{cm}$ portion of the distal clavicle [26].

The direct, or superior, arthroscopic technique utilizes a bursal-sparing approach, requiring a 2.7-mm arthroscope and mechanized burr to begin excision and a larger 4-mm arthroscope and instruments to complete the procedure [29]. This approach is ideal for patients with isolated AC joint pathology where exploration of the subacromial space is not required [22].

The indirect, or bursal, arthroscopic technique requires a bursectomy for visualization of the AC joint [26]. Considering most patients with AC joint pathology also suffer from some degree of impingement and subacromial pathology, the indirect approach becomes the more popular 


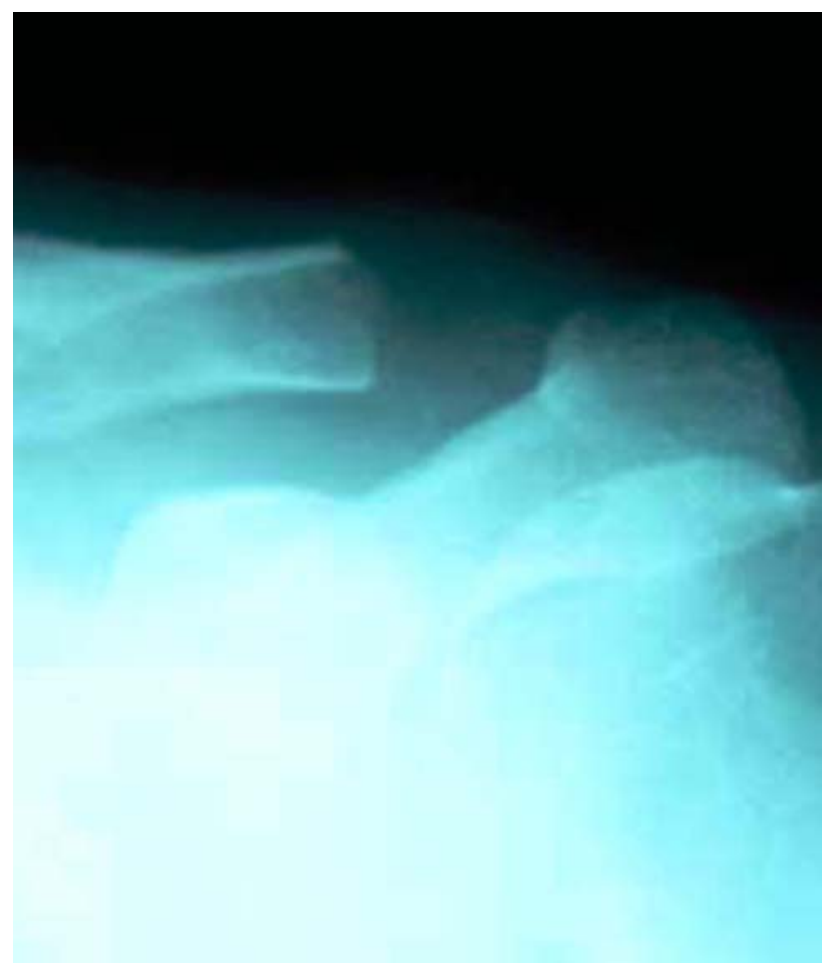

Fig. 6 Post-operative Zanca radiograph following arthroscopic distal clavicle resection. Image courtesy of Gregory N. Lervick, MD, Minnesota Sports Medicine

surgical choice [22]. This approach can be used for distal clavicle excision only, or in combination with acromioplasty and/or subacromial decompression, or rotator cuff repair $[26,29,30]$. An indirect technique also reduces the risk of post-operative instability of the clavicle by preserving the superior AC ligaments [22].

\section{Complications}

In an attempt to classify the complications of $\mathrm{AC}$ joint resection, Basmania et al. [31] performed an analysis of 42 patients who underwent open distal clavicle resection and determined inadequate resection, diagnostic errors, joint instability, and weakness accounted for the majority of problems. These complications have also been reported in arthroscopic techniques and are not limited to the open procedure [14].

Inadequate resection of the distal clavicle is also a common cause of persistent pain post-operatively [14]. Neer [32] reported inadequate resection of the posterior aspect of the distal clavicle during arthroscopic procedures can cause abutment with the acromion leading to pain. Inadequate resection is not a common complication of the open technique [33].

Diagnostic error plays a role in postoperative complications if $\mathrm{AC}$ joint pathology is only a partial contributing factor to a patient's shoulder pain. A diagnosis isolating the $\mathrm{AC}$ joint as the sole cause of shoulder pain is necessary before proceeding with a surgical treatment. Nuber and Bowen [22] state a lidocaine injection into the $\mathrm{AC}$ joint with $100 \%$ resolution can ensure a localized AC joint pathology. If pathology beyond the $\mathrm{AC}$ joint is suspected, the indirect arthroscopic technique can be utilized to allow a more thorough examination of the glenohumeral joint and subacromial space.

Joint instability can occur following damage to the superior and posterior portions of the AC ligaments which provide the maximum restraint to posterior motion of the clavicle [34]. Blazar et al. [35] noted anterior-posterior motion of the clavicle was increased by an average of $5.5 \mathrm{~mm}$, compared to a normal shoulder, following both open and arthroscopic techniques. Nuber and Bowen [22] state resection of larger amounts of the distal clavicle may disrupt the AC ligaments causing horizontal instability of the clavicle with abutment against the spine of the clavicle. Surgical approaches, such as the indirect arthroscopic technique, will avoid the more superior portions of the AC ligament and reduce the risk of joint instability.

Weakness of the shoulder following distal clavicle resection has been varied. Resultant weakness following an open technique, according to Shaffer [14], is due to the reattachment of the deltoid and trapezius muscles. Cook and Tibone [36] attribute the lack of strength to AC ligament injury. Martin et al. [37] found no weakness among 29 shoulders examined following indirect distal clavicle resection.

\section{Outcomes}

Patients are supported in a sling for a few days following both open and arthroscopic surgical techniques. Postoperative physical therapy with assisted exercises may begin within 2-5 days after surgery. While recovery time varies, patients can expect to return to activities within 2 3 months [22].

Literature demonstrates the open distal clavicle resection has yielded a return of $50-100 \%$ good or positive results, with an average of $76.3 \%$ [26]. Eskola et al. [38] found $72 \%$ of 73 patients who underwent an open technique to have had "good" or "satisfactory" results. Slawski and Cahill [39] report a 100\% satisfaction rate among 17 patients who underwent open distal clavicle excision for non-traumatic osteolysis.

An evaluation of 50 indirect distal clavicle resections performed by Snyder et al. [40] demonstrated the average amount of clavicle resection was $14.8 \mathrm{~mm}$, with 47 patients (94\%) reporting good to excellent results, 3 patients $(6 \%)$ reporting fair results, and $98 \%$ of patients reporting they were satisfied with the procedure. In an evaluation of 41 
patients who underwent direct arthroscopic distal clavicle resection by Zawadsky et al. [41], 22 cases (54\%) demonstrated excellent results, $16(39 \%)$ good results, and 3 (7\%) poor results due to continued instability of the distal clavicle. The study proposed a distal clavicular resection of 4-7 mm could yield good results.

\section{Summary}

The prominence of complaints related to shoulder pain demands an increased understanding of all clinical aspects related to the AC joint. Awareness of appropriate diagnostic techniques is necessary in localizing pathology to the AC joint. In the initial stages of treatment for AC joint osteoarthritis, attempts at non-surgical treatment modalities are recommended. All decisions regarding surgical intervention should (1) take place following the failure of nonsurgical treatment options and (2) take into account the need for further diagnostic evaluation. The open and direct techniques are both ideal for patients with isolated AC joint pathology where exploration of the subacromial space is not required, whereas the indirect arthroscopic approach can provide further evaluation of the subacromial space and an increased flexibility if further surgical repair beyond the AC joint is needed. Surgical complications, such as weakness of the deltoid and trapezius muscles and clavicular instability, should also be considered prior to deciding on the best surgical approach.

\section{References}

1. Urwin M, Symmons D, Allison T, Brammah T, Busby H, Roxby $\mathrm{M}$ et al. Estimating the burden of musculoskeletal disorders in the community: the comparative prevalence of symptoms at different anatomical sites, and the relation to social deprivation. Ann Rheum Dis. 1998;57:649-55.

2. Zanca P. Shoulder pain: involvement of the acromioclavicular joint-analysis of 1000 cases. Am J Roentgenol Radium Ther Nucl Med. 1971;112(3):493-506.

3. Strobel K, Pfirrman C, Zanetti M, Nagy L, Hodler J. MRI features of the acromioclavicular joint that predict pain relief from intraarticular injection. AJR. 2003;181:755-60.

4. Petersson CJ. Degeneration of the acromioclavicular joint: a morphological study. Acta Orthop Scand. 1983;54:434-8.

5. Horvath F, Kery L. Degenerative deformations of the acromioclavicular joint in the elderly. Arch Gerontol Geriatr. 1984;3:259-65.

6. Stein BE, Wiater JM, Pfaff HC, Bigliani LU, Levine WN. Detection of acriomioclavicular joint pathology in asymptomatic shoulders with magnetic resonance imaging. J Shoulder Elbow Surg. 2001;10:204-8.

7. Needell SD, Zlatkin MB, Sher JS, Murphy BJ, Uribe JW. MR imaging of the rotator cuff: peri-tendinous and bone abnormalities in an asymptomatic population. AJR. 1996;166:863-7.

8. Jacob AK, Sallay PI. Therapeutic efficacy of corticosteroid injections in the acromioclavicular joint. Biomed Sci Instrum. 1997;34:380-5.
9. McCluskey GM III, Todd J. Acromioclavicular joint injuries. J South Orthop Assoc. 1995;4:206-3.

10. Lervick G. Direct arthroscopic distal clavicle resection: a technical review. Iowa Orthop J. 2005;25:149-56.

11. Terry G, Chopp T. Functional anatomy of the shoulder. J Athl Train. 2000;35(3):248-55.

12. Donatelli R. Physical therapy of the shoulder. St. Louis: Elsevier Inc.; 2004.

13. Henry MH, Liu SH, Loffredo AJ. Arthroscopic management of the acromioclavicular joint disorder: a review. Clin Orthop Relat Res. 1995;316:276-83.

14. Shaffer BS. Painful conditions of the acromioclavicular joint. J Am Acad Orthop Surg.1999;7:176-88.

15. Taft TN, Wilson FC, Oglesby JW. Dislocation of the acromioclavicular joint: an end-result study. J Bone Joint Surg Am. 1987;69:1045-51.

16. Bergfeld JA, Andrish JT, Clancy WG. Evaluation of the acromioclavicular joint following first- and second-degree sprains. Am J Sports Med. 1978;6:153-9.

17. Buttaci CJ, Stitik TP, Yonclas PP, Foye PM. Osteoarthritis of the acromioclavicular joint: a review of anatomy, biomechanics, diagnosis, and treatment. Am J Phys Med Rehabil. 2004;83(10):791-7.

18. DePalma AF. The role of the disks of the sternoclavicular and the acromioclavicular joints. CORR. 1959;13:222-33.

19. Worcester JN, Green DP. Osteoarthritis of the acromioclavicular joint. Clin Orthop. 1968;58:69-73.

20. Garretson RB, Williams GR. Clinical evaluation of injuries to the acromioclavicular and sternoclavicular joints. Clin Sports Med. 2003;22:239-54.

21. Tallia AF, Cardone DA. Diagnostic and therapeutic injection of the shoulder region. Am Fam Physician. 2003;67(6):1271-8.

22. Nuber GW, Bowen MK. Arthroscopic treatment of acromioclavicular joint injuries and results. Clin Sports Med. 2003;22:30117.

23. Ernberg LA, Potter HG. Radiographic evaluation of the acromioclavicular and sternoclavicular joints. Clin Sports Med. 2003;22:255-75.

24. Shubin SBE, Ahmad CS, Pfaff C, Bigliani LU, Levine WN. A comparison of MRI findings of the acromioclavicular joint in symptomatic versus asymptomation patients. Presented at American Orthpaedic Society for Sports Medicine Meeting. Orlando, FL, June 30-July 3; 2002.

25. Alasaarela E, Tervonen O, Takalo R, Lahde S, Suramo I. Ultrasound evaluation of the acromioclavicular joint. J Rheumatol. 1997;24:1959-63.

26. Rabalais RD, McCarty E. Surgical treatment of symptomatic acromioclavicular joint problems. Clin Orthop Relat Res. 2006;455:30-7.

27. Codsi MJ (2007) The painful shoulder: when to inject and when to refer. 74(7):473-4, 477-8, 480-2, 485-8.

28. Lemos MJ, Tolo ET. Complications of the treatment of the acromioclavicular and sternoclavicular joint injuries, including instability. Clin Sports Med. 2003;22:371-85.

29. Sellards R, Nicholson GP. Arthroscopic distal clavicle resection. Oper Tech Sports Med. 2004;12:18-26.

30. Kay SP, Ellman H, Harris E. Arthroscopic distal clavicle excision: technique and early results. Clin Orthop Relat Res. 1994:301:181-4.

31. Basmania CJ, Wirth MA, Rockwood CA Jr, Moya D. Failed distal clavicle resection. Orthopaedic Trans. 1995;19:355.

32. Neer CS II. Shoulder reconstruction. Philadelphia, PA: WB Saunders; 1990.

33. Sachs RA, Stone MC, Devine S. Open vs. arthroscopic acromioplasty: a prospective randomized study. Arthroscopy. 1994;10:248-54. 
34. Klimkiewicz JJ, Williams GR, Sher JS, Karduna A, DesJardins JD, Iannotti JP. The acromioclavicular capsule as a restraint to posterior translation of the clavicle, biomechanical analysis. JSES. 1999;8:119-24.

35. Blazar PE, Iannotti JP, Williams GR. Anteroposterior instability of the distal clavicle after distal clavicle resection. Clin Orthop. 1998;348:114-20.

36. Cook FF, Tibone JE. The Mumford procedure in athletes: an objective analysis of function. Am J Sports Med. 1988;16:97-100.

37. Martin SD, Baumgarten TE, Andrews JR. Arthroscopic resection of the distal clavicle with simultaneous subacromial decompression. Orthop Trans. 1996;20:19-20.
38. Eskola A, Santavirta S, Viljakka HT, Wirta J, Partio TE, Hoikka $\mathrm{V}$. The results of operative resection of the lateral end of the clavicle. J Bone and Joint Surg Am. 1996;78:584-7.

39. Slawski DP, Cahill BR. Atraumatic osteolysis of the distal clavicle: results of open surgical excision. Am J Sports Med. 1994;22:267-71.

40. Snyder SJ, Banas MP, Karzel RP. The arthroscopic Mumford procedure: an analysis of results. Arthroscopy. 1995;11:157-64.

41. Zawadsky M, Marra G, Wiater JM, Levine WN, Pollock RG, Flatow EL et al. Osteolysis of the distal clavicle, long-term results of arthroscopic resection. Arthroscopy. 2000;16(6): $600-5$. 